\title{
THE INFLUENCE OF A MAGNETIC FIELD ON RADIATIVE DAMPING OF MAGNETOATMOSPHERIC OSCILLATIONS
}

\author{
DIPANKAR BANERJEE \\ Indian Institute of Astrophysics, Bangalore 560034, India. \\ Armagh Observatory, Armagh, BT61 9DG, N. Ireland ${ }^{\dagger}$ \\ S. S. HASAN \\ Indian Institute of Astrophysics, Bangalore 560034, India. \\ AND

\section{J. CHRISTENSEN-DALSGAARD} \\ Teoretisk Astrofysik Center, Danmarks Grundforskningsfond, and \\ Institut for Fysik og Astronomi, Aarhus Universitet, \\ DK-8000 Aarhus C, Denmark
}

\begin{abstract}
We investigate the influence of a magnetic field on the radiative damping of magnetoatmospheric waves, extending our previous work on the adiabatic modes of an isothermal stratified atmosphere with a uniform vertical magnetic field. Banerjee, Hasan \& Christensen-Dalsgaard $(1996,1997)$ generalized this work to include radiative effects using Newton's law of cooling for a weak magnetic field. The present study examines the variation of the mode damping rate with increasing magnetic field strength. We find that a moderate field suppresses radiative damping.
\end{abstract}

Key words: MHD, magnetic fields, atmosphere, oscillations

\section{Introduction}

It is now generally accepted on the basis of observations (Stenflo 1989) that the solar magnetic field is highly structured, with most of the magnetic flux in the photosphere being clumped into intense flux tubes with kilogauss field strengths. Flux tube diameters vary from a few hundred kilometers in small-scale tubes to several thousand

${ }^{\dagger}$ Present address 
kilometers in sunspots. Oscillations in a fairly broad range of frequencies have been reported in magnetic flux tubes (Moore \& Rabin 1985). More recently MHD oscillations have been observed in the solar photosphere (Norton et al. 1997) and active regions (Horn \& Staude 1997). The purpose of this contribution is to examine whether radiative damping is important in intense flux tubes.

The present investigation is a continuation of earlier work by Hasan \& ChristensenDalsgaard (1992) and Banerjee, Hasan \& Christensen-Dalsgaard (1995), who examined the effects of a vertical magnetic field on the normal adiabatic modes of an isothermal stratified atmosphere by combining a semi-analytic approach, based on asymptotic dispersion relations, with numerical solutions. These results were extended by Banerjee et al. (1997) to include radiative dissipation using Newton's law of cooling for a weak magnetic field. In the present work we examine the variation of the mode damping rate with magnetic field strength.

\section{Magnetic modes in an isothermal radiative atmosphere}

The radiativly damped normal modes of an isothermal atmosphere with a vertical magnetic field have been discussed in detail by Banerjee (1997). We consider a cavity of vertical extent $d$ and look for standing-wave solutions. We introduce a dimensionless wave number $K=k / H$ and dimensionless frequency $\Omega=\omega H / c_{s}$, where $H$ is the scale height of the atmosphere and $c_{s}$ is the sound speed. We characterize the radiative losses by a dimensionless constant radiative cooling time scale

$$
\tilde{\tau}_{R}=\frac{c_{s} \rho c_{V}}{16 H \chi \sigma T^{3}}
$$

where $\chi$ is the mean absorption coefficient per unit length, $c_{V}$ is the specific heat at constant volume and $\sigma$ is the Stefan-Boltzman constant. Banerjee (1997) derived a dispersion relation which allows the effect of a weak magnetic field on the modes to be studied. It was shown that the normal-mode frequency can be expressed as a sum of an elementary mode frequency and a first-order correction, due to coupling between different modes. Asymptotic analysis revealed that in the weak- and strongfield limits, the modes are essentially decoupled into magnetic and $p$-type modes.

We focus on the magnetic modes and study the radiative damping of these modes as the field strength is increased. For the adiabatic case, the magnetic modes have frequencies (in the weak-field limit) $\Omega_{m} \propto n \epsilon$, where $n$ denotes the mode order and $\epsilon=v_{A, 0} / c_{s},\left(v_{A, 0}\right.$ being the photospheric Alfven speed at the lower boundary).

To illustrate the effect of the magnetic field strength on the damping of the magnetic modes, Fig. 1 shows the variation of the imaginary part of frequency (which is a measure of the damping) with $\epsilon$ for fixed $K=0.3, \tilde{\tau}_{R}=0.5$ and $D=d / H=1.0$. These results were generated by solving the MHD equations numerically assuming rigid boundary conditions at the top and bottom boundaries. The various line styles corresponds to different order magnetic modes. It is evident that as we increase the value of $\epsilon$ (increasing magnetic field strength) the imaginary part of $\Omega$ decreases, indicating a reduction in the damping of the wave modes. In the limit of a very strong field, the damping rate becomes negligibly small. The non-monotonic behaviour of the curves in Fig. 1 is due to mode interaction, which leads to the occurrence of avoided crossings when the frequencies of different modes become almost equal. Also, the damping of the modes is enhanced at these crossings (Banerjee et al. 1996, 1997). Our conclusion that the damping rate decreases with field strength is in qualitative agreement with the work of Bogdan \& Knölker (1989), who examined a similar 


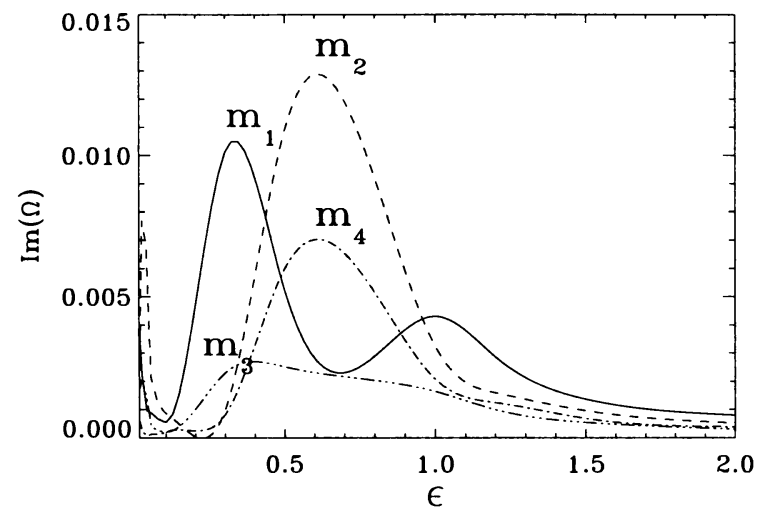

Figure 1. Variation of the imaginary part of $\Omega$ with $\epsilon$ for $K=0.3, \tilde{\tau}_{R}=0.5$ and $D=1.0$ for various order magnetic modes, shown in different line styles.

problem for an unstratified atmosphere. They argued that a uniform magnetic field reduces the temperature perturbations associated with MHD waves and therefore suppresses the radiative damping of these disturbances. A similar result was found for a horizontal magnetic field by Bünte \& Bogdan (1994).

\section{Umbral oscillations}

We now consider the importance of radiative effects in damping umbral oscillations in sunpots. For simplicity we assume that the umbral atmosphere is isothermal and extends over several scale heights. It is well known that periods of oscillations above a sunspot's umbra range between 100 and $200 \mathrm{~s}$. Extensive measurements in different spectral lines have been made (Thomas et al. 1984, 1987; Alisandrakis et al. 1992). Kentischer \& Mattig (1995) recently observed umbral oscillations in the middle and upper chromosphere, with pronounced power at frequencies between 5 and $9 \mathrm{mHz}$. They concluded that the first peak at $5.9 \mathrm{mHz}$ consists mainly of upward propagating waves traveling at the sound speed. There are indications that these waves have their origin in the photospheric resonator. The second distribution is located at $7.5 \mathrm{mHz}$ and involve standing waves which are confined to the chromosphere (chromospheric resonator). Previous studies (Hasan 1991; Wood 1990, 1997) have shown that the the waves in the chromosphere are essentially slow magnetoacoustic modes or $p$-modes. The frequencies of the slow magnetoacoustic modes or $p$-modes with radiative damping for $K=0$ are given by (Banerjee 1997),

$$
\Omega_{p, n}=\sqrt{\tilde{\gamma}\left(\frac{n^{2} \pi^{2}}{D^{2}}+\frac{1}{4}\right)}
$$

where $n$ is the order and $\tilde{\gamma}=\left(1+i \Omega \tilde{\tau}_{R} \gamma\right) /\left(\gamma+\mathrm{i} \Omega \tilde{\tau}_{R} \gamma\right), \gamma$ being the adiabatic exponent.

Table 1 gives the eigenfrequencies of different order $p$-modes for an isothermal atmosphere with $D=10, \tilde{\tau}_{R}=0.5$ and $\epsilon=0.84(B \sim 2 \mathrm{kG})$. The relation between the horizontal wave number and the radius of the flux tube was discussed by Banerjee (1997). It is evident from Table 1 that radiative cooling introduces a positive imaginary 


\begin{tabular}{cccccccccc}
\hline & \multicolumn{2}{c}{$\begin{array}{c}\text { Nearly adiabatic } \\
\text { case } \\
\left(\tilde{\tau}_{R}=100\right)\end{array}$} & & \multicolumn{2}{c}{$\begin{array}{c}\text { Radiative case } \\
\left(\tilde{\tau}_{R}=0.5\right)\end{array}$} & \multicolumn{4}{c}{$\begin{array}{c}\text { Nearly isothermal } \\
\text { case } \\
\left(\tilde{\tau}_{R}=0.05\right)\end{array}$} \\
\cline { 2 - 10 } Mode & $\operatorname{Re}(\Omega)$ & $\operatorname{Im}(\Omega)$ & $\mathrm{P}(\mathrm{s})$ & $\operatorname{Re}(\Omega)$ & $\operatorname{Im}(\Omega)$ & $\mathrm{P}(\mathrm{s})$ & $\operatorname{Re}(\Omega)$ & $\operatorname{Im}(\Omega)$ & $\mathrm{P}(\mathrm{s})$ \\
\hline$p_{1}$ & 0.5903 & 0.0007 & 164 & 0.5203 & 0.0658 & 186 & 0.458 & 0.0125 & 211 \\
$p_{2}$ & 0.8030 & 0.0009 & 120 & 0.7075 & 0.0895 & 136 & 0.624 & 0.0170 & 155 \\
$p_{3}$ & 1.0700 & 0.0013 & 90 & 0.9400 & 0.1190 & 103 & 0.828 & 0.0227 & 117 \\
\hline
\end{tabular}

TABLE 1. Eigenfrequencies of different order $p$-modes for a model atmosphere with $D=10, B=2 \mathrm{kG}, \tilde{\tau}_{R}=0.5$ and $K=0.1$.

component of the eigenfrequencies, corresponding to comparatively small damping. The computed frequencies match very well with those calculated from expression (2). Furthermore, the $p_{2}$-mode has a period of $136 \mathrm{~s}$ in the presence of radiative losses, which is very close to the observed peak at $7.5 \mathrm{mHz}$ (period $134 \mathrm{~s}$ ) by Kentischer et al. (1995). This observation was reported at layers near the temperature minimum which correspond to about $1000 \mathrm{~km}$ over the photosphere $(D=10$, in our notation).

In summary, we have examined the influence of the magnetic field on wave damping in an isothermal stratified atmosphere. We have shown that as the field strength is raised, the damping rate decreases. We have applied our calculations to umbral oscillations in sunspots and demonstrated that they exhibit modest damping, even when the radiative cooling time is comparable with the pulsation period.

This work was partly supported by the Danish National Research Foundation through the establishment of the Theoretical Astrophysics Center. and the Smithsonian Institution through a foreign currency grant.

\section{References}

Alisandrakis, T. E., Georgakilas, A. A. , Dialetis, D., 1992, Solar Phys., 138, 93

Banerjee, D., 1997, Ph.D Thesis, Bangalore University, India

Banerjee, D., Hasan, S. S., Christensen-Dalsgaard, J., 1995, Astrophys. J. 451, 825

Banerjee, D., Hasan, S. S., Christensen-Dalsgaard, J., 1996, Bull. Astron. Soc. India 24, 325

Banerjee, D., Hasan, S. S., Christensen-Dalsgaard, J., 1997, Solar Phys., 172, 53

Bünte, M., Bogdan, T. J., 1994, Astron. Astrophys. 283, 642

Bogdan, T. J., Knölker, M., 1989, Astrophys. J. 339, 579

Hasan, S. S., 1991, Astrophys. J. 366, 328

Hasan, S. S. Christensen-Dalsgaard, J., 1992, Astrophys. J. 396, 311

Horn, T., Staude, J., 1997, these proceedings

Kentischer, T. J., Mattig, W., 1995, Astron. Astrophys. 300, 539

Moore, R. L., Rabin, D., 1985, Ann. Rev. Astron. Astrophys. 23, 239

Norton, A., Ulrich, R. K., Bogart, R. S., Bush, R. I., Hoeksema, J. T., 1997, these proceedings Stenflo, J. O., 1989, Astron. Astrophys. Rev 1, 3

Thomas, J. H., Cram, L. E., Nye, A. H., 1984, Astrophys. J. 285, 368

Thomas, J. H., Lites, B. W., Gurman, J. B., Ladd, E. F., 1987, Astrophys. J. 312, 457

Wood, W. P., 1990, Mon. Not. Rol. Astr. Soc., 251, 427

Wood, W. P., 1997, Sol. Phys., 173, 259 